\title{
Dynamic Elimination of Overflow Tests in a Trace Compiler
}

\author{
Rodrigo Sol ${ }^{1}$, Christophe Guillon ${ }^{2}$, Fernando Magno Quintão Pereira ${ }^{1}$, \\ and Mariza A.S. Bigonha ${ }^{1}$ \\ 1 UFMG - 6627 Antônio Carlos Av, 31.270-010, Belo Horizonte, Brazil \\ $\{$ rsol,fpereira, mariza\}@dcc.ufmg.br \\ 2 STMicroelectronics - 12 Jules Horowitz St, B.P. 217, 38019, Grenoble, France \\ christophe.guillon@st.com
}

\begin{abstract}
Trace compilation is a technique used by just-in-time (JIT) compilers such as TraceMonkey, the JavaScript engine in the Mozilla Firefox browser. Contrary to traditional JIT machines, a trace compiler works on only part of the source program, normally a linear path inside a heavily executed loop. Because the trace is compiled during the interpretation of the source program the JIT compiler has access to runtime values. This observation gives the compiler the possibility of producing binary code specialized to these values. In this paper we explore such opportunity to provide an analysis that removes unnecessary overflow tests from JavaScript programs. Our optimization uses range analysis to show that some operations cannot produce overflows. The analysis is linear in size and space on the number of instructions present in the input trace, and it is more effective than traditional range analyses, because we have access to values known only at execution time. We have implemented our analysis on top of Firefox's TraceMonkey, and have tested it on over 1000 scripts from several industrial strength benchmarks, including the scripts present in the top 100 most visited webpages in the Alexa index. We generate binaries to either $\mathrm{x} 86$ or the embedded microprocessor ST40-300. On the average, we eliminate $91.82 \%$ of the overflows in the programs present in the TraceMonkey test suite. This optimization provides an average code size reduction of $8.83 \%$ on ST40 and $6.63 \%$ on x86. Our optimization increases TraceMonkey's runtime by $2.53 \%$.
\end{abstract}

\section{Introduction}

JavaScript is the most popular programming language used in the client-side of web applications [12. Supporting this statement is the fact that JavaScript is used in 97 out of the 100 most popular websites in the alexa 2010 report1. Thus, it is very important that JavaScript programs benefit from efficient execution environments. Web browsers normally interpret programs written in this language. However, to achieve execution efficiency, JavaScript programs can be

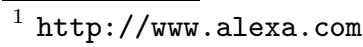

J. Knoop (Ed.): CC 2011, LNCS 6601, pp. 2 21, 2011.
(c) Springer-Verlag Berlin Heidelberg 2011 
compiled during interpretation - a process called just-in-time (JIT) compilation. There are many ways to perform JIT compilation. TraceMonkey [14, the Mozilla Firefox 3.1's JIT compiler, translates the most executed program traces into machine code. A program trace is a linear sequence of code representing a path inside the program's control flow graph.

The Firefox JIT compiler, among many optimizations, does type specialization on JavaScript programs. For instance, JavaScript sees numbers as double precision 64-bit values in the IEEE 754 format [8, p.29]; however, TraceMonkey tries to manipulate them as integer values every time it is possible. Dealing with integers is much faster than handling floating-point arithmetics, but it is necessary to ensure that this optimization does not change the semantics of the program. So, every time an operation produces a result that might exceed the precision of the integer type, it is necessary to perform an overflow test. In case the test fails, float-point numbers must replace the original integer operands.

Overflow tests are pervasive in the machine code produced by TraceMonkey, a performance nuisance already acknowledged by the Mozilla community 2 . Overflow tests impose two major problems. First, because each test may force an early exit from tracing mode, these tests complicate optimizations that require code motion, such as partial redundancy elimination and instruction scheduling. Second, instructions that handle overflows increase code size: about $12.3 \%$ of the $\mathrm{x} 86$ code produced by TraceMonkey per script in this compiler's test suite implement overflow tests. This extra code is a complication on small devices that run JavaScript, such as the ST family of microcontroller 3 . We have attacked this issue via a flow sensitive range analysis that proves that some overflow tests are redundant, and we present the results of this work in this paper. Our analysis runs in linear time on the number of instructions in the input trace. This analysis is implemented on top of TraceMonkey; however, it does not depend on a particular compiler. On the contrary, it works on any JIT engine that uses the trace paradigm to do code generation.

Our algorithm does range analysis 1720 , i.e, it tries to estimate the lowest and greatest values that can be assigned to any variable. However, our approach differs from previous works because we use values known only at runtime in order to put bounds on the range of values that integer variables might assume during the program execution. This is a form of partial evaluation [18, yet done at runtime 4], because our analysis is invoked by a just-in-time compiler while the target application is being interpreted. By relying on such values we are able to perform much more aggressive range inferences than traditional static analyses. In terms of implementation, our analysis is similar to the ABCD algorithm that eliminates array bound-checks [3]; however, there are important differences. First, our algorithm is simpler, for it runs on a program trace, which is straight-line code. Because it runs on a trace, our algorithm is linear on the number of program variables, and not quadratic, as other analyses that keep def-use chains of variables. Second, we perform the whole analysis at once, in a

${ }^{2}$ https://bugzilla.mozilla.org/show_bug.cgi?id=536641

${ }^{3}$ http://www.st.com/mcu/familiesdocs -51 .html 
single traversal of the input trace, whereas ABCD runs on demand. In addition to these differences, the fact that we use the runtime value of variables lets us be less conservative.

We have implemented our analysis on top of TraceMonkey (release from 201010-3), the JIT compiler used by the Firefox web browser, and we have targeted two different processors, x86 and ST40. We have correctly compiled and run over one million lines of JavaScript code taken from a vast collection of benchmarks, including the TraceMonkey's test suite, and the top 100 webpages according to the Alexa index. Currently our implementation can only remove overflow tests from arithmetic operations performed on variables declared locally, i.e, we do not handle global variables yet. Nevertheless, we recognize $59 \%$ of the overflow tests in the TraceMonkey test suite, which contains over 800 scripts. On average, our algorithm eliminates $91.82 \%$ of the overflow tests that we recognize in these scripts, providing an average code size reduction of $6.63 \%$ on $\mathrm{x} 86$, and $8.83 \%$ on ST40. Our research-quality implementation adds $2.53 \%$ of time overhead on the core TraceMonkey implementation (time to compile and run the script) without other optimizations enabled. However, we speculate that an industrial-strength implementation of our algorithm will be able to obtain runtime gains. We have instrumented the Firefox browser, to check the behavior of our algorithm in actual webpages: on the average we remove $53.5 \%$ of the overflow tests per webpage. This number is lower when compared to the TraceMonkey's test suite because there are more global variables in actual webpages.

The remainder of this paper is organized as follows. Section 2 describes the TraceMonkey JIT compiler. In that section we show, by means of a simple example, how a trace is produced and represented. We describe our analysis in Section 3. Section 4 provides some experimental data that shows that our analysis is effective. We discuss related work in Section 5. Finally, Section 6 concludes this paper.

\section{TraceMonkey in a Nutshell}

There exists a number of recent works describing the implementation of tracebased JIT compilers, such as Tamarim-trace 6], HotpathVM [15], Yeti 27] and TraceMonkey [14]. In order to explain this new compilation paradigm, in this section we describe the TraceMonkey implementation. TraceMonkey has been built on top of SpiderMonkey, the original JavaScript interpreter used by the Firefox Browser. To produce x86 machine code from JavaScript sources, TraceMonkey uses the Nanoji 4 compiler. The whole compilation process goes through three intermediate representations, a path that we reproduce in Figure 1 .

1. AST: the abstract syntax tree that the parser produces from a script file.

2. Bytecodes: a stack based instruction set that SpiderMonkey interprets.

3. LIR: the low-level three-address code instruction representation that Nanojit receives as input.

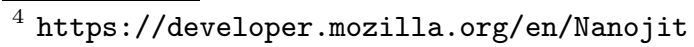




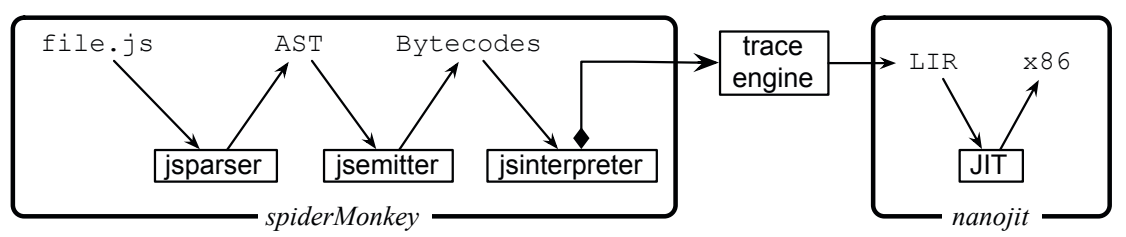

Fig. 1. The TraceMonkey JavaScript JIT compiler

SpiderMonkey has not been originally conceived as a just-in-time compiler, a fact that explains the seemly excessive number of intermediate steps between the source program and the machine code. Segments of LIR instructions - a trace in TraceMonkey's jargon - are produced according to a very simple algorithm [14]:

1. each conditional branch is associated to a counter initially set to zero.

2. If the interpreter finds a conditional branch during program interpretation, then it increments the counter. The process of checking and incrementing counters is called, in TraceMonkey's jargon, the monitoring phase.

3. If the counter is two or more, and no trace exists for that counter, then the trace engine starts translating the bytecodes to a segment of LIR instructions, while they are interpreted. Overflow tests are inserted into this LIR segment. The process of building the trace is called recording phase.

4. Once the trace engine finds the original branch that started the recording process, the current segment is passed to Nanojit.

5. The Nanojit compiler translates the LIR segment, including the overflow tests, into machine code, which is dumped into main memory. The program flow is diverted to this code, and direct machine execution starts.

6. After the machine code runs, or in case an exceptional condition happens, e.g, an overflow test fails or a branch leaves the trace, the flow of execution goes back to the interpreter.

From bytecodes to LIR: we use the program in Figure 2 (a) to illustrate the process of trace compilation, and also to show how our analysis works. This is an artificial program, clearly too naive to find use in the real world; however, it contains the subtleties necessary to put some strain on the cheap analysis that must be used in the context of a just-in-time compiler. This program would yield the bytecode representation illustrated in Figure 2 (b). Notice that we took the liberty of simplifying the bytecode intermediate language used by TraceMonkey.

A key motivation behind the design of the analysis that we present in Section 3 is the fact that TraceMonkey might produce traces for program paths while these paths are visited for the first time. This fact is a consequence of the algorithm that TraceMonkey uses to identify traces. At the beginning of the interpretation 


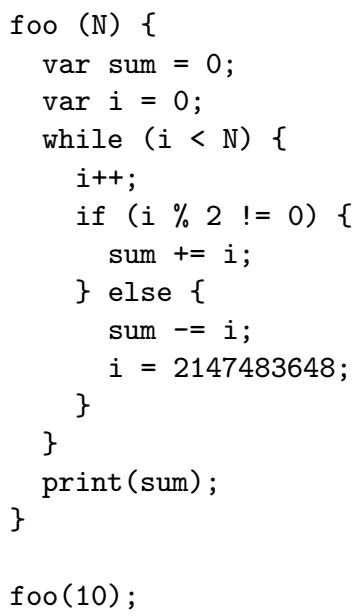

(a)

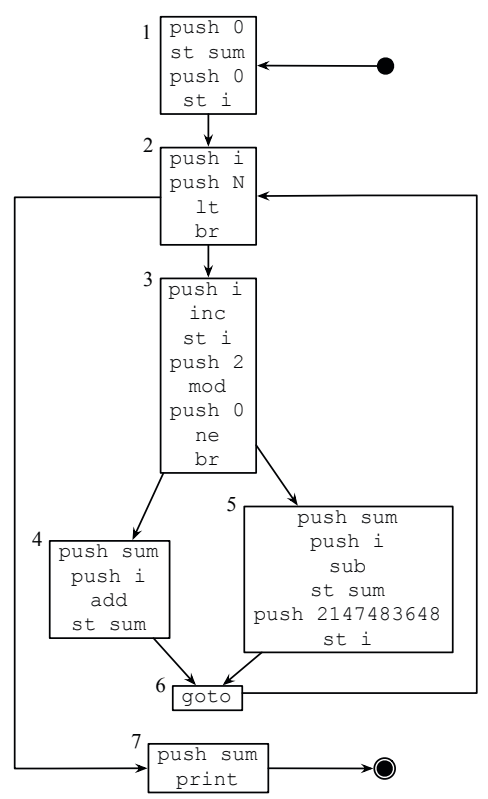

(b)

Fig. 2. Example of a small JavaScript program and its bytecode representation

process TraceMonkey finds the branch at Basic Block 2 in Figure 2 (b), and the trace engine increments the counter associated to that branch. The next branch will be found at the end of Basic Block 3, and this branch's counter will be also incremented. The interpreter then will find the goto instruction at the end of Basic Block 6, which will take the program flow back to Block 2. At this moment the trace engine will increment again the counter of the branch in that basic block. Once the trace engine finds a counter holding the value two, it knows that it is inside a loop, and starts the recording phase, which produces a LIR segment. However, this segment does not correspond to the first part of the program visited: in the second iteration of the loop, Basic Block 5 is visited instead of Basic Block 4. In this case, the segment that is recorded is formed by Basic Blocks 2, 3, 5 and 6.

Because the trace that is monitored by the trace engine is not necessarily the trace that is recorded into a LIR segment, it is difficult to remove overflow tests during the recording phase. A conditional test, such as $a<N$, where $N$ is constant, helps us to put bounds on the range of values that $a$ might assume. However, in order to know that $N$ is constant, we must ensure that the entire recorded segment does not contain commands that change $N$ 's value. Although it is not possible to remove overflow tests directly during the recording phase, it is possible to collect constraints on the ranges of the variables in this step. These constraints will be subsequently used to remove overflow tests at the 


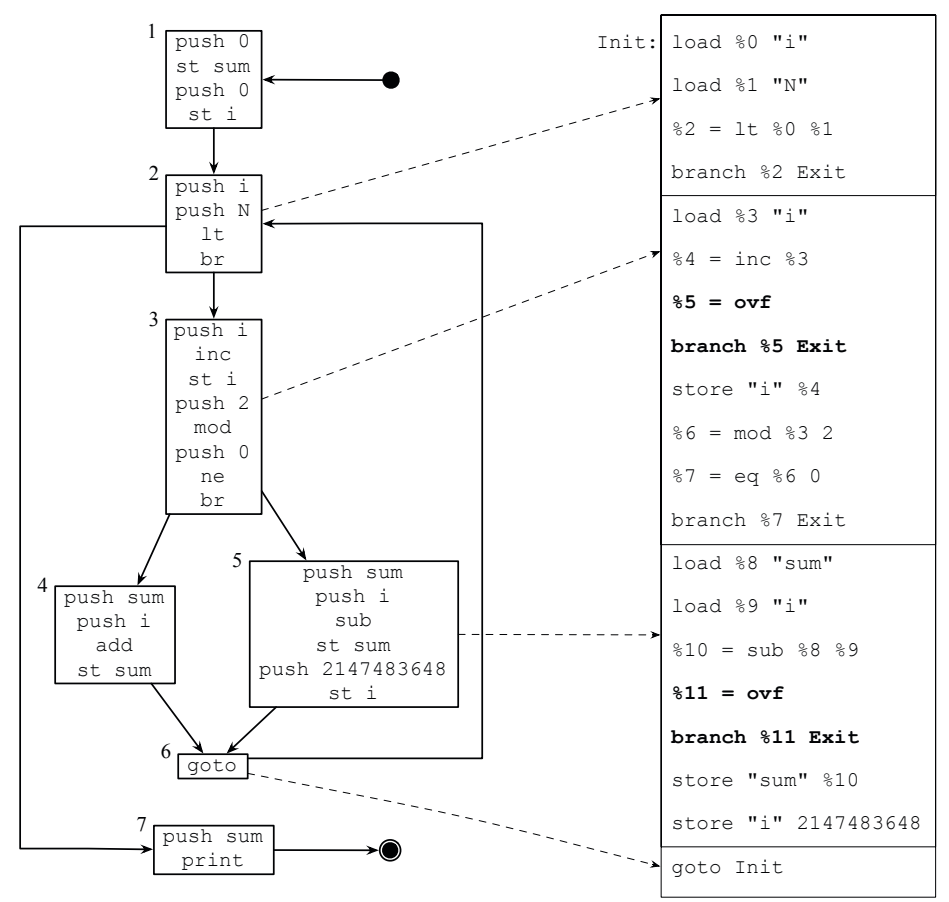

Fig. 3. This figure illustrates the match between the recorded trace and the LIR segment that the trace engine produces for it

Nanojit level. Thus, we perform the elimination of overflow tests once the whole LIR segment has been produced, right before it is passed to Nanojit. Continuing with our example, Figure 3 shows the match between the recorded trace and the LIR segment that the trace engine produces for it.

From LIR to $x 86$ : Before passing the LIR segment to Nanojit, the interpreter augments this segment with two sequences of instructions: the prologue and the epilogue. The prologue contains code to map the values from the interpreter's execution environment to the execution environment where the compiled binary will run. The epilogue contains code to perform the inverse mapping. Nanojit produces machine code, e.g x86, ARM, ST40, from the LIR segment that it receives from the interpreter. The overflow tests are implemented as side-exit code: a sequence of instructions that has two functions: (i) it recovers the state of the program right before the overflow took place, and (ii) it jumps to the exit of the trace, returning control back to the interpreter. Figure 4 shows a simplified version of the $\mathrm{x} 86$ code that would be produced for our example trace. Notice that our analysis has no role at this point of the compilation process we include it in this discussion only to give the reader a full picture of the trace compiler. 
(LIR)

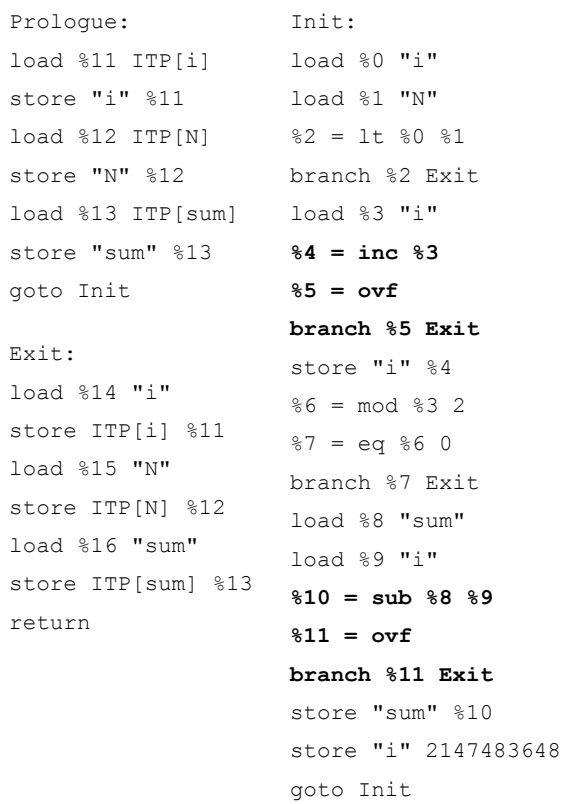

(x86)

$\begin{array}{cccc}\begin{array}{c}\text { Prologue } \\ \text { pushl }\end{array} & \ldots & \text { RS1 : } & \\ \text { movl } & \ldots & \operatorname{movl} & \ldots \\ \text { movl } & \ldots & \operatorname{movl} & \ldots \\ \text { movl } & \ldots & \operatorname{movl} & \ldots \\ \text { jmp } & \text { Init } & \text { jmp } & \text { Exit } \\ \text { Init: } & & & \\ \text { leal } & \ldots & \text { Rs2 : } & \\ \text { incl } & \ldots & \operatorname{movl} & \ldots \\ \text { jvf } & \text { Rs1 } & \operatorname{movl} & \ldots \\ \text { movl } & \ldots & \operatorname{movl} & \ldots \\ \text { andl } & \ldots & \operatorname{movl} & \ldots \\ \text { testb } & \ldots & \text { jmp } & \text { Exit }\end{array}$

je Exit

movl ...

leal ...

subl ...

jvf RS2

movl ...

movl ...

cmpl ...

jb Init

Exit:

movl ...

movl ...

movl ...

popl ...

leave

Fig. 4. The LIR code, after the insertion of a prologue and an epilogue, and the schematic $\mathrm{x} 86$ trace produced by Nanojit

\section{Flow Sensitive Range Analysis}

The flow sensitive range analysis that we use to remove overflow tests relies on a directed acyclic graph to determine the ranges of the variables. This graph, henceforth called constraint graph, has four types of nodes:

Name: represent program variables. Each node contains a counter, which represents a particular definition of a variable. These nodes are bound to an integer interval, which represents the range of values that the definition they encode might assume.

Assignment: denoted by mov, represent the copy of a value to a variable.

Relational: represent comparison operations, which are used to put bounds on the ranges of the variables. The comparison operations considered are: equals (eq), less than (lt), greater than (gt), less than or equals (le), and greater than or equals (ge).

Arithmetic: represent operations that might require an overflow test. We have two types of arithmetic nodes: binary and unary. The binary operations are addition (add), subtraction (sub), and multiplication (mul). The unary operations are increment (inc) and decrement (dec). Division is not handled because it might legitimately produce floating-point results. 
The analysis proceeds in two phases: construction of the constraint graph and range propagation. The remaining of this section describes these phases.

\subsection{Construction of the Constraint Graph}

We build the constraint graph during the trace recording phase of TraceMonkey, that is, while the instructions in the trace are being visited and a LIR segment is being produced. In order to associate range constraints to each variable in the source program we use a program representation called Extended Static Single Assignment (e-SSA) [3] form, which is a superset of the well known SSA form [9]. In the e-SSA representation, a variable is renamed after it is assigned a value, or after it is used in a conditional.

Converting a program to e-SSA form requires a global view of the program, a requirement that a trace compiler cannot fulfill. However, given that we are compiling a program trace, that is, a straight line segment of code, the conversion is very easy, and happens at the same time that the constraint graph is built, e.g, during the trace recording step. The conversion works as follows: counters are mantained for every variable. Whenever we find a use of a variable $v$ we rename it to $v_{n}$, where $n$ is the current value of the counter associated to $v$. Whenever we find a definition of a variable we increment its counter. Considering that the variables are named after their counters, incrementing the counter of a variable effectively creates a new name definition in our representation. So far our renaming is just converting the source program into Static Single Assignment form [9]. The e-SSA property comes from the way that we handle conditionals. Whenever we find a conditional, e.g, $a<b$, we learn new information about the ranges of $a$ and $b$. Thus, we redefine $a$ and $b$, by incrementing their counters.

There are two events that change the bounds of a variable: simple assignments and conditional tests. The first event determines a unique value for the variable. The second puts a bound in one of the variable's limits, lower or upper. These are the events that cause us to increment the counters associated to variables. Thus, it is possible to assign unique range constraints to each new definition of a variable. These range constraints take into consideration the current value of the variables at the time the variable is found by the trace engine. We determine these values by inspecting the interpreter's stack. We have designed the following algorithm to build the constraint graph:

1. initialize counters for every variable in the trace. We do this initialization on the fly: the first time a variable name is seen we set its counter to zero, otherwise we increment its current counter. If we see a variable for the first time, then we mark it as input, otherwise we mark it as auxiliary. If a variable is marked as input, then we set its upper and lower limits to the value that the interpreter currently holds for it. Otherwise, we set the variable boundaries to undefined values, e.g, ] $-\infty,+\infty[$.

2. For each instruction $i$ that we visit:

(a) if $i$ is a relational operation, say $v<u$, we build a relational node that has two predecessors: variable nodes $v_{x}$ and $u_{y}$, where $x$ and $y$ are counters. This node has two successors, $v_{x+1}$ and $u_{y+1}$. 


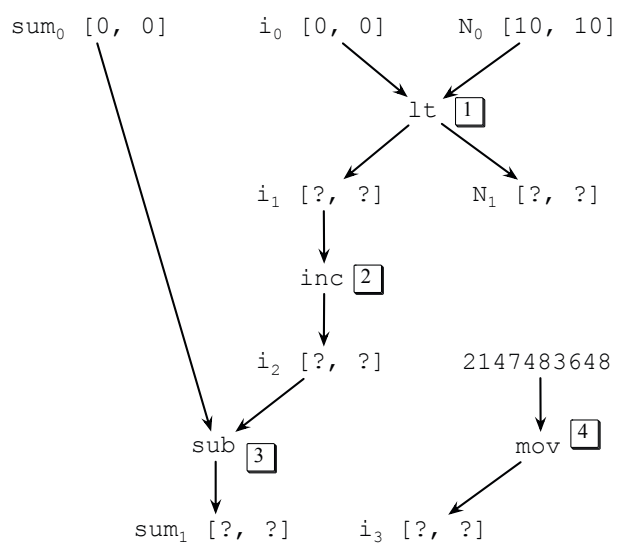

Fig. 5. Constraint graph for the trace in Figure 3. The numbers in boxes denote the order in which the nodes were created.

(b) For each binary arithmetic operation, e.g, $v=t+u$, we build an arithmetic node $n$. Let the nodes related to variables $t_{x}$ and $u_{y}$ be the predecessors of $n$, and let $v_{z}$ be its successor.

(c) For each unary operation, say $u++$, we build a node $n$, with one predecessor $u_{x}$, and one successor $u_{x+1}$.

(d) For each copy assignment, e.g, $v=u$, we build an assignment node, which has predecessor $u_{x}$, and successor $v_{y+1}$, assuming $y$ is $v$ 's counter.

Figure 5 shows the constraint graph to our running example, assuming that the function foo was called with the parameter $N=10$. Notice that we bound the input variables to intervals: sum $_{0} \subseteq[0,0], i_{0} \subseteq[0,0]$ and $N_{0} \subseteq[10,10]$. When constructing the constraint graph, it is important to maintain a list with the order in which each node was created. This list, which we represent by the numbers in boxes, will be later used to guide the range propagation phase.

\subsection{Range Propagation}

During the range propagation phase we find which overflow tests are necessary, and which ones can be safely removed from the target code. The propagation of ranges is preceded by a trivial initialization step, when we replace the constraints of the input variables with ] $-\infty,+\infty$ [ if those variables have been updated inside the trace. This is the case, for example, of variables sum ${ }_{0}$ and $i_{0}$ in the example from Figure 5. In order to do the propagation of range intervals, we visit all the arithmetic and relational nodes, in topological order. This ordering is given by the "age" of the node. Nodes that have been created earlier, during the construction of the constraint graph are visited first. Notice that we get this ordering for free, simply storing the arithmetic and relational nodes in a queue while we create them, during the construction of the constraint graph. If every variable is defined before being used, then by following the node creation order, 


\begin{tabular}{|c|c|}
\hline \multicolumn{2}{|c|}{ Arithmetics } \\
\hline$x+(+\infty)=+\infty+x=+\infty, x \neq-\infty$ & $x+(-\infty)=-\infty+x=-\infty, x \neq+\infty$ \\
\hline$x \times( \pm \infty)= \pm \infty \times x= \pm \infty, x>0$ & $x \times( \pm \infty)= \pm \infty \times x=\mp \infty, x<0$ \\
\hline \multicolumn{2}{|c|}{ Increment: $x_{1}=x_{0}+1$} \\
\hline$\underline{x_{0} \cdot l+1>\text { MAX_INT }}$ & $\underline{x_{0} \cdot u+1>\text { MAX_INT }}$ \\
\hline$x_{1} . l=+\infty$ & $x_{1} \cdot u=+\infty$ \\
\hline$\underline{x_{0} . l+1 \leq \text { MAX_INT }}$ & $\underline{x_{0} \cdot u+1 \leq \text { MAX_INT }}$ \\
\hline$x_{1} \cdot l=x_{0} . l+1$ & $x_{1} \cdot u=x_{0} \cdot u+1$ \\
\hline \multicolumn{2}{|c|}{ Addition: $x=a+b$} \\
\hline$\underline{a . l+b . l<\text { MIN_INT }}$ & $a . u+b . u<$ MIN_INT \\
\hline$x . l=-\infty$ & $x \cdot u=-\infty$ \\
\hline$\underline{a . l+b . l>\text { MAX_INT }}$ & $a . u+b . u>$ MAX_INT \\
\hline$x . l=+\infty$ & $x . u=+\infty$ \\
\hline$\underline{\text { MIN_INT } \leq a . l+b . l \leq \text { MAX_INT }}$ & MIN_INT $\leq a . u+b . u \leq$ MAX_INT \\
\hline$x . l=a . l+b . l$ & $x . u=a . u+b . u$ \\
\hline \multicolumn{2}{|c|}{ Multiplications: $x=a \times b$} \\
\hline$\underline{a . l \times b . l<\text { MIN_INT }}$ & $\underline{a . u \times b . u<\text { MIN_INT }}$ \\
\hline$x . l=-\infty$ & $x . u=-\infty$ \\
\hline$a . l \times b . l>$ MAX_INT & $a . u \times b . u>$ MAX_INT \\
\hline$x . l=+\infty$ & $x=$ \\
\hline$\underline{\mathrm{MIN} \_I N T} \leq a . l \times b . l \leq \mathrm{MAX} \_\mathrm{INT}$ & $\underline{\text { MIN_INT } \leq a . u \times b . u \leq \text { MAX_INT }}$ \\
\hline$x . l=\operatorname{MIN}(a .\{l, u\} \times b \cdot\{l, u\})$ & $x \cdot u=\operatorname{MAX}(a \cdot\{l, u\} \times b \cdot\{l, u\})$ \\
\hline
\end{tabular}

Fig. 6. Range propagation for arithmetic nodes. Decrements and subtractions are similar to increments and additions. We use $a .\{l, u\} \times b .\{l, u\}$ as a short form for $(a . l \times b . l, a . l \times b . u, a . u \times b . l, a . u \times b . u)$.

the propagation of ranges guarantees that whenever we reach an arithmetic, relational or assignment node, all the name nodes that point to it have been visited before.

Each arithmetic and relational node causes the propagation of ranges in a particular way, always preserving the invariant that the lower bound of an interval is less than or equal its upper bound. Figure 6 shows the updating rules that we use for some arithmetic nodes. We use standard IEEE extended arithmetics [16], except that, to improve our analysis, we assume that the result of multiplying infinity and zero is zero. Furthermore, we let the sum of $]-\infty, x[$ plus $]+\infty,+\infty[$ to be $]-\infty,+\infty[$, and vice-versa. We denote the interval $[l, u]$ associated to a 


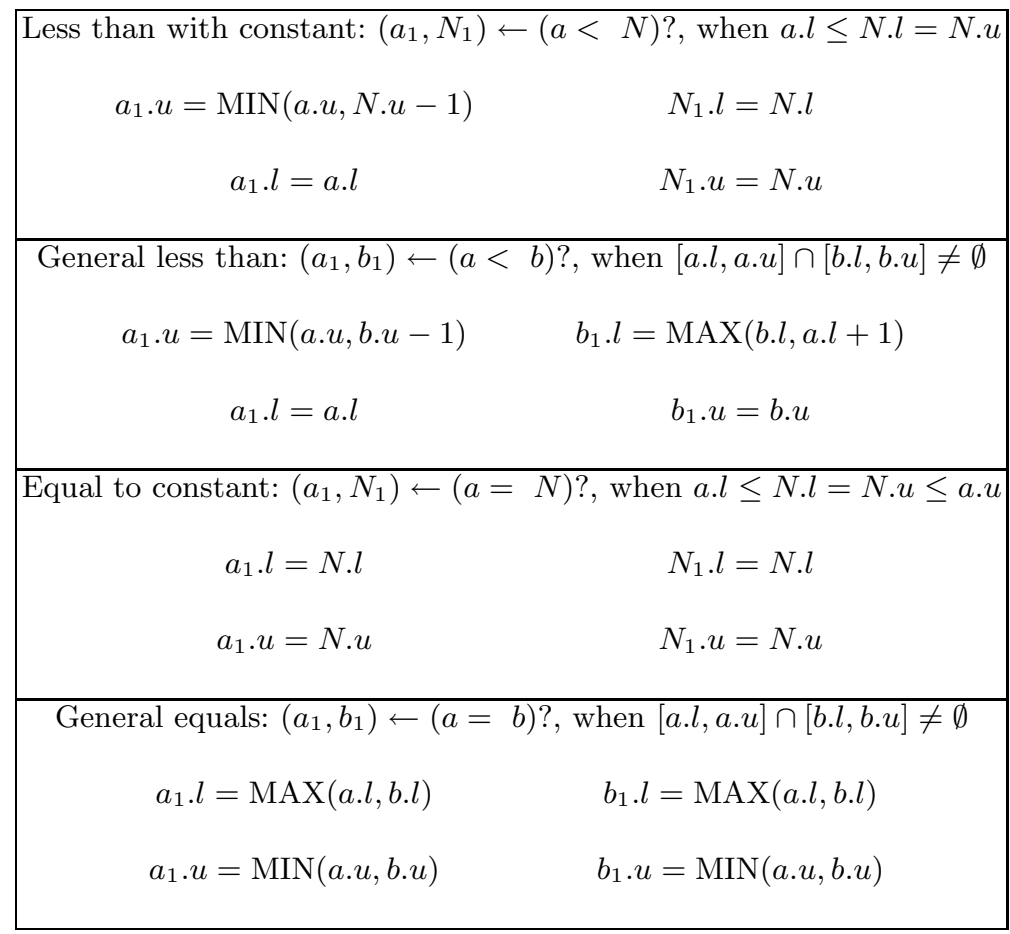

Fig. 7. Range propagation for two relational nodes. The value of variable $N$ is not updated inside the trace.

node $a$ by $a . l$ and a.u. Although we state the range propagation rules using infinity precision arithmetics, our actual implementation uses wrapping semantics, i.e: if $x+1>x$, then $x=+\infty$. Only arithmetic nodes might cause overflows; and each of our five types of arithmetic nodes might produce overflows in different ways. For instance, if we find an increment of $[-\infty, v . u]$, then we keep the overflow associated to this node, as long as $v \cdot u+1 \leq$ MAX_INT. Figure 7 shows the updating rules for some relational nodes. These nodes are not associated to any overflow test, but they help us to constrain the range of intervals bound to program variables. As an optimization, we do not update the ranges of variables that are not defined inside the trace. This is the case, for instance, of Variable $N$ in the program of Figure 2 (a). Notice that we do not perform range updates that break the invariant $v . l \leq v . u$.

After range propagation we have a conservative estimate of the intervals that each integer variable might assume during program execution. This information allows us to go over the LIR segment, before it is passed to Nanojit, removing the overflow tests that our analysis has deemed unnecessary. Figure 8 shows this step: we have removed the overflow test from the inc operation, because it receives as input a node bound to the interval ] $-\infty, 9]$; hence, its result will never be greater than 10. However, our analysis cannot prove that the test in the sub operation is also unnecessary, although that is the case. 


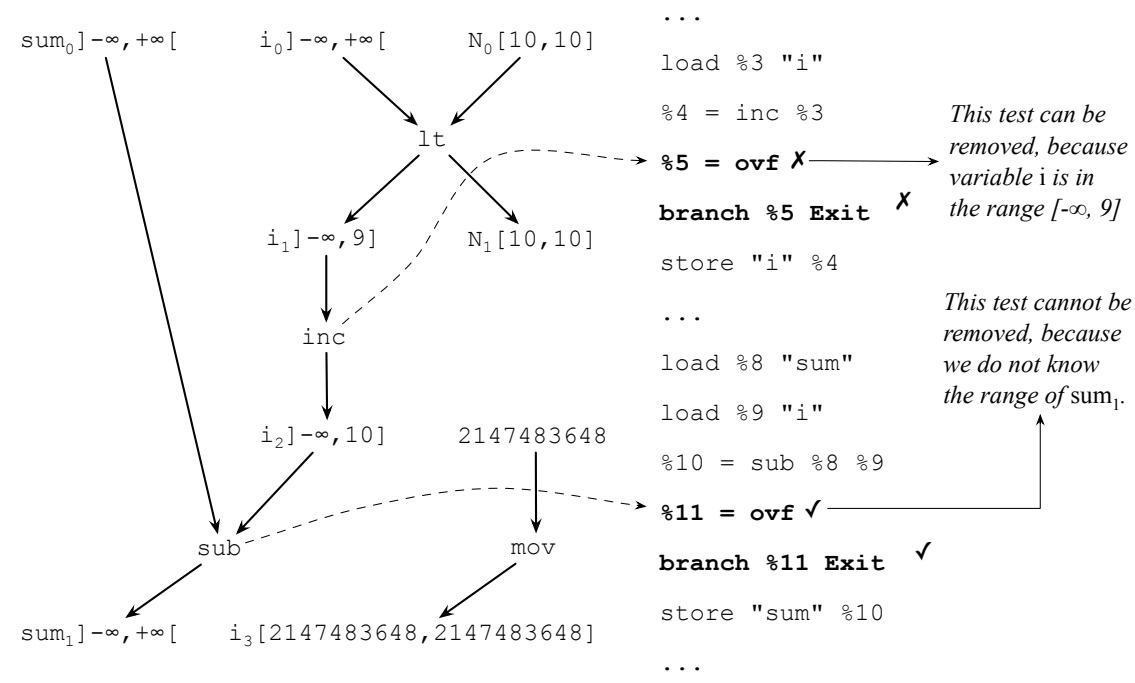

Fig. 8. Once we know the ranges of each variable involved in an arithmetic operation we can remove the associated overflow test, if it is redundant

\subsection{Complexity Analysis}

The proposed algorithm has running time linear on the number of instructions of the source trace. To see this fact, notice that the constraint graph has a number of conditional and arithmetic nodes proportional to the number of instructions in the trace. During our analysis we traverse the constraint graph one time, at the range propagation phase, visiting each node only once. We do not have to preprocess the graph beforehand, in order to sort it topologically, because we get this ordering from the sequence in which instructions are visited in the source trace. Our algorithm is also linear in terms of space, because each type of arithmetic node has a constant number of predecessors and successors. This low complexity is in contrast to the complexity of many graph traversal algorithms, which are $O(E)$, where $E$ is the number of edges in the graph. These algorithms have a worst case quadratic complexity on the number of vertices, because $E=$ $O\left(V^{2}\right)$. We do not suffer this drawback, for in our case $E=O(V)$.

\section{Experimental Results}

We have implemented our algorithm on top of TraceMonkey, revision 201010-01. Our implementation handles the five arithmetic operations described in Section 3.1, namely additions, subtractions, increments, decrements and multiplications. We perform the range analysis described in Section 3 during the recording phase of TraceMonkey, that is, while a segment of JavaScript bytecodes is translated to a segment of LIR. We have also modified Nanojit to remove the overflow tests, given the results of our analysis. Our current implementation 
has some shortcomings, which are all due to our limited understanding of TraceMonkey's implementation, and that we are in the process of overcoming:

- we cannot read values stored in global variables, a fact that hinders us from removing overflows related to operations that manipulate these values.

- We cannot recognize when TraceMonkey starts tracing constructs such as foreach $\{\ldots\}$, while (true) $\{\ldots\}$ and loops that range on iterators.

The benchmarks. We have correctly compiled and executed over one million lines of JavaScript code that we took from three different benchmark suites:

Alexa top 100: the 100 most visited webpages, according to the Alexa index 5 . This list includes Google, Facebook, Youtube, etc. We tried to follow Richards et al.'s methodology [12], manually visiting each of these pages with our instrumented Firefox. Notice that we present results for only 80 of these benchmarks, because we did not find overflow tests in 20 webpages.

Trace-Test: the test suite that is used in TraceMonkey6. This collection of scripts includes popular benchmarks, such as Webkit's Sunspider and Google's V8. Many scripts do not contain arithmetic operations; thus, we show results only for the 224 scripts that contains at least one overflow test.

PeaceKeeper: an industrial strength benchmark used to test browsers.

The hardware. We have used our modified TraceMonkey compiler in two different hardware: (i) x86: $2 \mathrm{GHz}$ Intel Core 2 Duo, with $2 \mathrm{~GB}$ of RAM, featuring Mac OS 10.5.8. (ii) ST40-300: a real-time processor manufactured by STMicroelectronics. The ST40 runs STLinux 2.3 (kernel 2.6.32), has a $450 \mathrm{MHz}$ clock, provides $200 \mathrm{MB}$ of physical memory and $512 \mathrm{MB}$ of virtual memory. This target is a two level cache architecture with separated $32 \mathrm{~KB}$ intruction and $32 \mathrm{~KB}$ data L1 caches on top of a $256 \mathrm{~KB}$ unified L2 cache.

How effective is our algorithm? Figure 9] shows the effectiveness of our algorithm when we run it on the Alexa webpages and the Trace-Test scripts. We have ordered the scripts on the $\mathrm{x}$-axis according to the effectiveness of our algorithm. We present static numbers, in the sense that we have not instrumented the binary traces to see how many times each overflow test is executed. On the average, we remove $91.82 \%$ of the overflows tests from the scripts in Trace-Test, and $53.50 \%$ of the overflow tests from the Alexa webpages. This is the geometric mean; that is, we are very effective in removing overflow tests from most of the scripts. In particular, we remove most of the overflow tests used in counters that index simple loops. However, the arithmetic mean is much lower, because of the outliners. Three of these scripts, which are part of SunSpider (included in TraceTest) deal with cryptography and manipulate big numbers. They account for 8,756 tests, out of which we removed only 347 . In absolute terms we remove 961

\footnotetext{
${ }^{5}$ http://www.alexa.com/

${ }^{6}$ http://hg.mozilla.org/tracemonkey/file/c3bd2594777a/js/src/ trace-test/tests

${ }^{7}$ http://service.futuremark.com/peacekeeper/index.action
} 

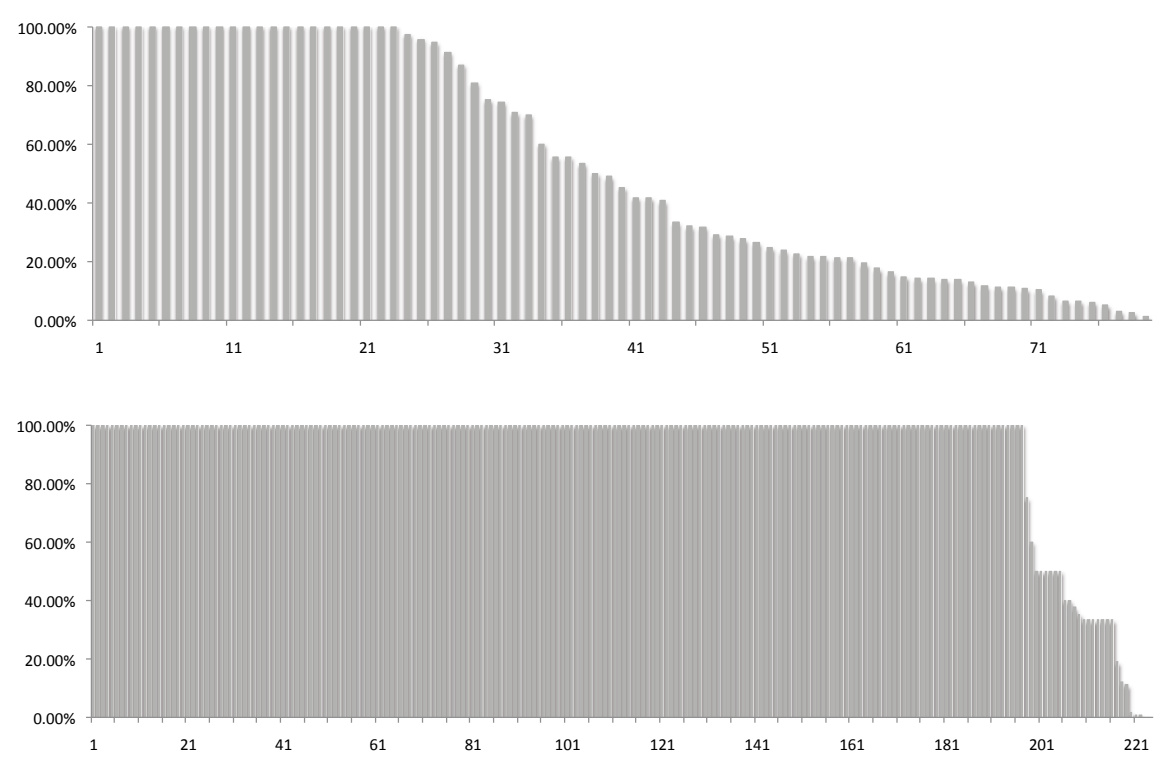

Fig. 9. The effectiveness of our algorithm in terms of percentage of overflow tests removed per script. (Top) Alexa; geo mean: 53.50\%. (Bottom) Trace-test; geo mean: $91.82 \%$. Hardware: same results for ST40 and x86. The 224 scripts are sorted by average effectiveness. We removed 700/859 overflow tests for PeaceKeeper (one script).

out of 11,052 tests in the Trace-Test benchmark, and 2,680 out of 11,126 tests in the Alexa benchmark. The arithmetic mean, in this case, is $8.7 \%$ for TraceTest, and $24.08 \%$ for Alexa. Figure 9 does not contain a chart for PeaceKeeper, because this benchmark contains only one script. We removed 700 tests out of the 859 tests that we found in this script.

What is the code size reduction due to the elimination of overflow tests? On the x86 target, TraceMonkey uses eight instructions to implement each overflow test, which includes the branch-if-overflow instruction, load instructions to reconstruct the interpreter state and an unconditional jump back to interpreter mode. On the ST40 microprocessor, TraceMonkey uses 10 instructions. We eliminate all these instructions after removing an overflow test. Figure 10] shows the average size reduction that our algorithm obtains on each target. On the average, we shrink each script by $8.83 \%$ on the ST40, and $6.63 \%$ on the $\mathrm{x} 86$. On most of the scripts the size reduction is modest; however, there are cases in which our algorithm decreases the binaries by over $60.0 \%$. Notice that by using integers instead of floating-point numbers TraceMonkey already reduces the size of the binaries that it produces. For instance, on the ST40, TraceMonkey obtains an average $31.47 \%$ of size reduction with the floating-point to integer optimization.

What is the effect of our algorithm on TraceMonkey's runtime? Figure 11 shows that, on the average, our algorithm has increased the runtime of 

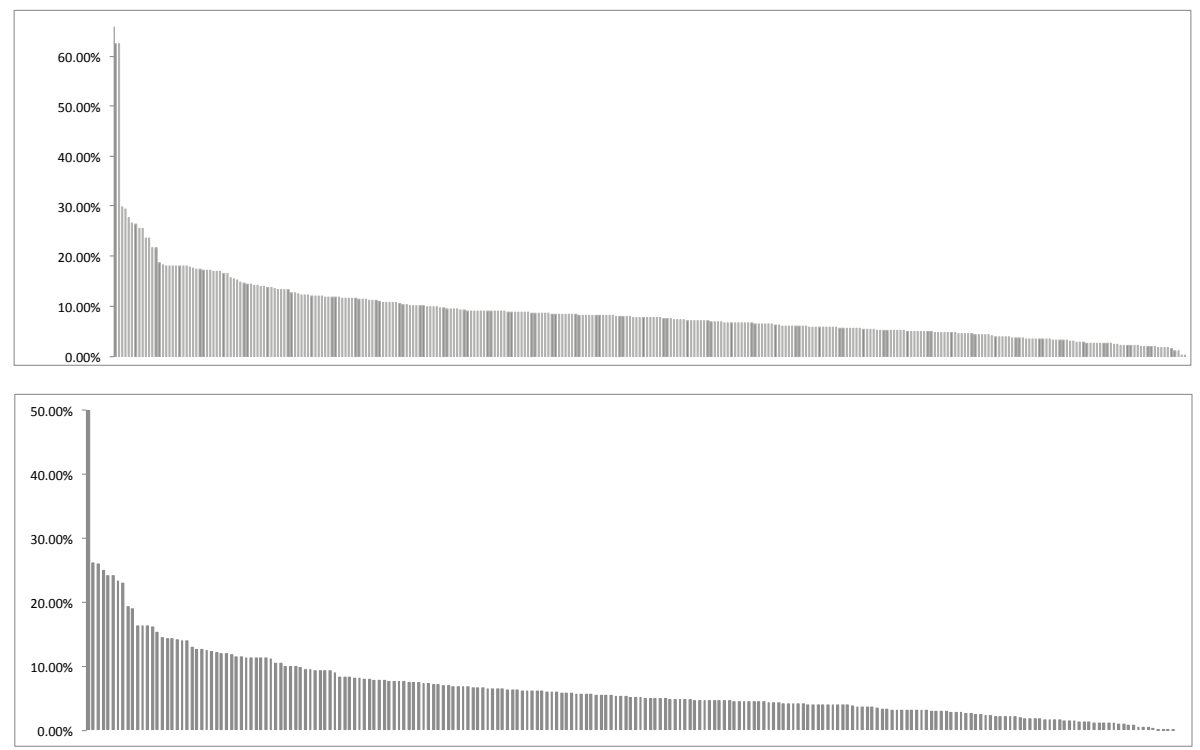

Fig. 10. Size reduction per script in two different architectures. (Top) ST40; geo mean: $8.83 \%$. (Bottom) x86; geo mean: 6.63\% Benchmark: Trace-Test. The 224 scripts are sorted by average size reduction.

TraceMonkey by $2.56 \%$ on the $\mathrm{x} 86$. This time includes parsing, interpreting, JIT compiling and executing the scripts. Our algorithm increases the time of JIT compilation, but decreases the time of script execution. So far the cost, in time, is negative for two reasons: a prototype implementation and a low benefit optimization. Figure 11 shows that running our analysis without disabling overflows tests increases TraceMonkey's runtime by $4.12 \%$. Furthermore, the effects of avoiding the overflows tests are negligible. Most of the time, the impact of an overflow test is the cost of executing the x86 instruction branch-if-overflow; however, this instruction is normally predicted as not taken. If we disable every overflow test without running the analysis, then we improve the runtime by $1.56 \%$ on the scripts that finish correctly. The noise in the chart is due to short runtimes - the slowest script executes for $0.73 \mathrm{~s}$, and the fastest for $0.01 \mathrm{~s}$.

Why sometimes we fail to remove overflows? We have performed a manual study on the 42 smallest programs from Trace-Test in which we did not remove overflow tests. Each of these tests contains a loop indexed by an induction variable. Figure 12 explains our findings. In $69 \%$ of the traces, we failed to remove the test because the induction variable was bounded by a global variable. As we explained before, our implementation is not able to identify the values of global variables; hence, we cannot use them in the estimation of ranges. In $17 \%$ of the remaining cases the trace is produced after a foreach control structure, which our current implementation fails to recognize. Once we fix these omissions, we will be able to remove at least one more overflow test in 36 out of these 42 


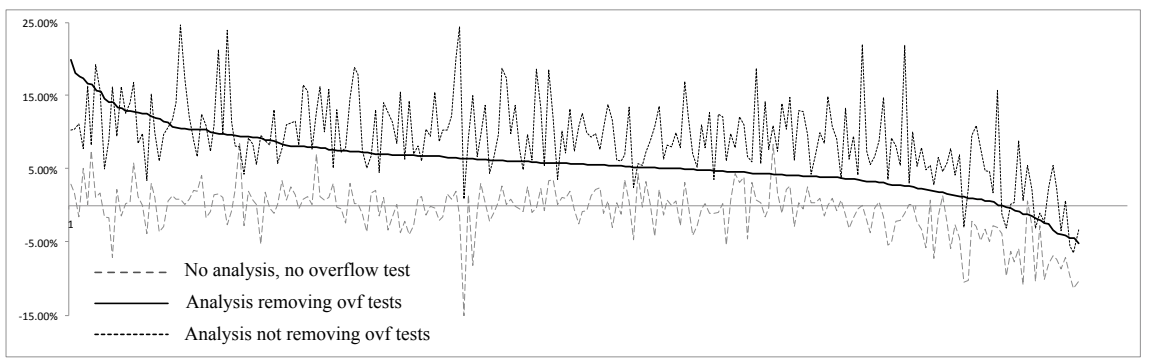

Fig. 11. Impact of our algorithm on TraceMonkey's runtime. Benchmark: Trace-Test. Hardware: x86. The 224 scripts are sorted by average runtime increase. We run each test 8 times, ignoring smallest and largest outliers. Zero is TraceMonkey's runtime without our analysis and with overflow tests enabled.

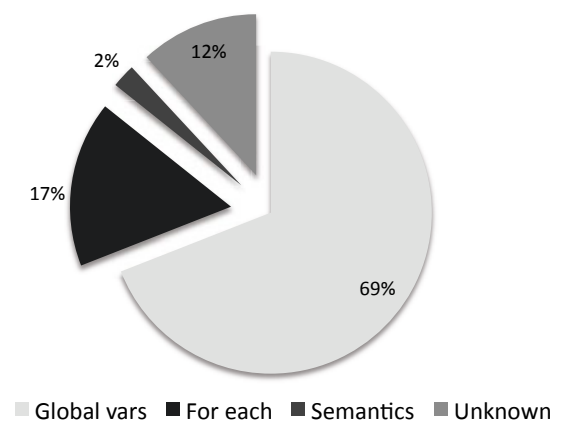

Fig. 12. The main reasons that prevent us from removing overflow tests

scripts analyzed. There was only one script in which our algorithm legitimately failed to remove a test: in this case the semantics of the program might lead to a situation in which an overflow, indeed, happens.

What we gain by knowing the runtime value of variables? We perform more aggressive range estimations than previous range analyses described in the literature, because we are running alongside a JIT compiler, a fact that gives us the runtime value of variables, including loop limits. Statically we can only rely on constants to start placing bounds in variable ranges. Non-surprisingly, a static implementation of our algorithm removes only 472 overflow tests from the Trace-Test, which corresponds to $37 \%$ of the tests that we remove dynamically.

Why are effectiveness results so different for Trace-Test and Alexa? Figure 9 shows that our algorithm is substantially more effective when applied on the programs in Trace-Test than on the programs in the Alexa top 100 pages. This happens because most of the scripts in the Trace-Test benchmark suite are small, and contain only simple loops controlled by locally declared variables, which our algorithm can recognize. In order to compare the two test suites, we have used our instrumented Firefox to produce the histogram of assembly 


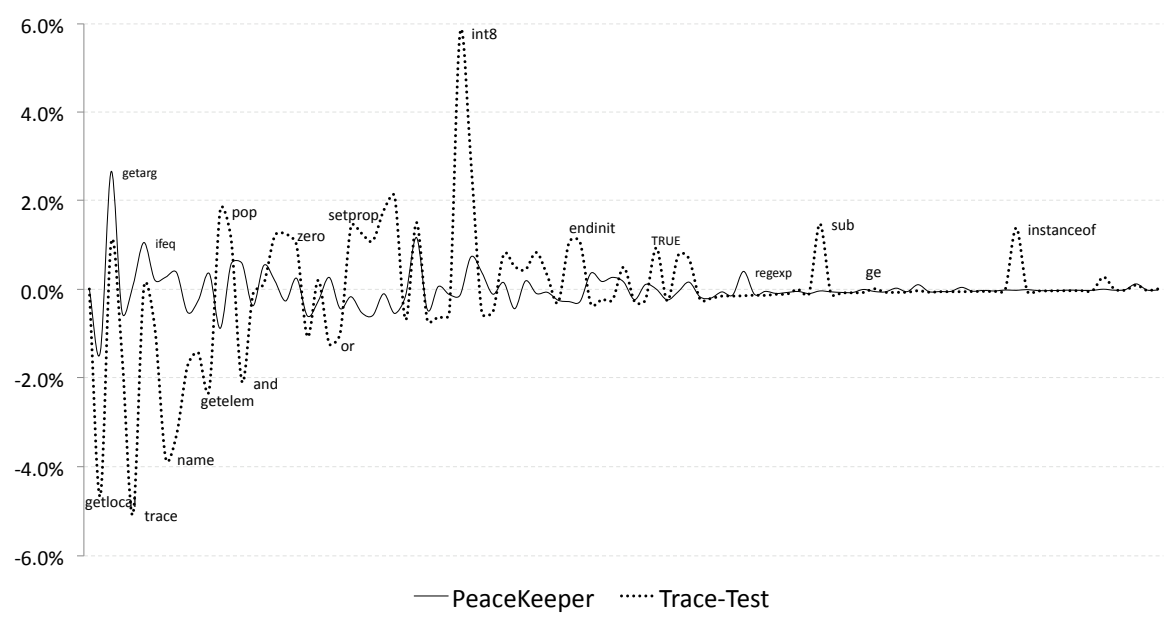

Fig. 13. A histogram of the bytecodes present in Trace-Test and PeaceKeeper, compared to the bytecodes present in the Alexa collection. X-axis: instructions $i$ ordered by their frequency in the Alexa collection.

instructions that TraceMonkey generates for each benchmark. Figure 13 shows the results of our findings. We have produced this chart by plotting pairs $(i, f(i))$, which we define as follows:

1. let $I$ be the list formed by the 100 most common SpiderMonkey bytecodes found in the Alexa benchmark. We order the bytecodes $i$ according to $a(i)$, the frequency of occurrences of $i$ in the Alexa collection.

2. For each bytecode $i \in I$ and benchmark $B \in\{$ Trace-Test, PeaceKeeper $\}$, we let $b(i)$ be $i$ 's frequency in $B$, and we let $f(i)=a(i)-b(i)$.

The closer to zero the values of $f(i)$, more similar is the frequency of $i$ in the Alexa collection and in the other benchmark. Using this rough criterion, we see that PeaceKeeper is closer to Alexa than Trace-Test. Summing up the absolute values of $f(i)$, we find 0.24 for PeaceKeeper and 0.75 for Trace-Test. Coincidentally, our analysis is more effective on Trace-Test than on PeaceKeeper.

\section{Related Work}

Just-in-time compilers are old allies of programming language interpreters. Since the seminal work of John McCarthy 19, the father of Lisp, a multitude of JIT compilers have been designed and implemented. A comprehensive survey on just-in-time compilation is given by John Aycock [1].

The optimization that we propose in this paper is a type of partial evaluation at runtime. Partial evaluation is a technique in which a compiler optimizes a program, given a partial knowledge of its input [18. Variations of partial evaluation have been used to perform general code optimization 22 23. This type of partial evaluation in which the JIT compiler uses runtime values to produce better 
binaries is sometimes called specialization by need [21]. Implementations that use this kind of technique include Python's Psyco JIT compiler [21, Matlab [710] and Maple 4. Partial evaluation has been used in the context of just-in-time compilation mostly as a form of type specialization [7514. That is, once the compiler proves that a value belongs into a certain type, it uses this type directly, instead of resorting to costly boxing and unboxing techniques.

The competition between popular browsers has brought renewed attention to trace compilation. However, the idea of focusing the compilation effort on code traces, instead of whole methods, has been known at least since the work of Bala et al. [2], or even before if we consider trace scheduling [1]. Traces have been independently integrated on JIT-compilers by Gal 13 , and Zaleski [27. Currently, there are many trace-based JIT-compilers 6141527. For a formal overview of trace compilation, we recommend the work of Guo and Palsberg [26].

Our algorithm to remove overflow tests is a type of range analysis [1720]. Range analysis tries to infer lower and upper bounds to the values that a variable might assume during the program execution. In general these algorithms rely on theorem provers, an approach deemed too slow to a JIT compiler. Bodik et al. 3] have described a specialization of range analysis that removes array bound checks, the ABCD algorithm, that targets JIT compilation. Zhendong and Wagner [25] have described a type of range analysis that can be solved in polynomial time. Stephenson et al. 24] have used a polynomial time analysis to infer the bitwidth of each integer variable used in the source program. Contrary to our approach, all these previous algorithms work on the static representation of the source program. Such fact severely constraints the amount of information that these analysis can rely on. By knowing the runtime value of program variables we can perform a very extensive, yet fast, range analysis.

\section{Conclusion}

This paper has presented a new algorithm to remove redundant overflow tests during the JIT compilation of JavaScript programs. The proposed algorithm works in the context of a trace compiler. Our algorithm is able to find very precise ranges for program variable by inspecting their runtime values. We have implemented our analysis on top of TraceMonkey, the JIT compiler used by the Mozilla Firefox browser to speed up the execution of JavaScript programs. We have submitted our implementation to Mozilla, as a Firefox patch, available at http://github.com/rodrigosol/Dynamic-Elimination-Overflow-Test. In terms of future work, we would like to improve the performance and effectiveness of our implementation. For instance, currently we can only read the runtime values of local variables, a limitation that we are working to overcome.

Acknowledgments. This project has been made possible by the cooperation FAPEMIG-INRIA, grant 11/2009. Rodrigo Sol is supported by the Brazilian Ministry of Education under CAPES and CNPq. We thank David Mandelin from the Mozilla Foundation for helping us with TraceMonkey. 


\section{References}

1. Aycock, J.: A brief history of just-in-time. ACM Computing Surveys 35(2), 97-113 (2003)

2. Bala, V., Duesterwald, E., Banerjia, S.: Dynamo: a transparent dynamic optimization system. In: PLDI, pp. 1-12. ACM, New York (2000)

3. Bodik, R., Gupta, R., Sarkar, V.: ABCD: eliminating array bounds checks on demand. In: PLDI, pp. 321-333. ACM, New York (2000)

4. Carette, J., Kucera, M.: Partial evaluation of maple. In: PEPM, pp. 41-50. ACM, New York (2007)

5. Chambers, C., Ungar, D.: Customization: optimizing compiler technology for self, a dynamically-typed object-oriented programming language. SIGPLAN Not. 24(7), 146-160 (1989)

6. Chang, M., Smith, E., Reitmaier, R., Bebenita, M., Gal, A., Wimmer, C., Eich, B., Franz, M.: Tracing for web 3.0: trace compilation for the next generation web applications. In: VEE, pp. 71-80. ACM, New York (2009)

7. Chevalier-Boisvert, M., Hendren, L., Verbrugge, C.: Optimizing Matlab through just-in-time specialization. In: Gupta, R. (ed.) CC 2010. LNCS, vol. 6011, pp. 46-65. Springer, Heidelberg (2010)

8. ECMA Committe. ECMAScript Language Specification. ECMA, 5th edn. (2009)

9. Cytron, R., Ferrante, J., Rosen, B.K., Wegman, M.N., Zadeck, F.K.: Efficiently computing static single assignment form and the control dependence graph. TOPLAS 13(4), 451-490 (1991)

10. Elphick, D., Leuschel, M., Cox, S.: Partial evaluation of MATLAB. In: Pfenning, F., Macko, M. (eds.) GPCE 2003. LNCS, vol. 2830, pp. 344-363. Springer, Heidelberg (2003)

11. Fisher, J.A.: Trace scheduling: A technique for global microcode compaction. Trans. Comput. 30, 478-490 (1981)

12. Richards, G., Lebresne, S., Burg, B., Vitek, J.: An analysis of the dynamic behavior of javascript programs. In: PLDI, pp. 1-12 (2010)

13. Gal, A.: Efficient Bytecode Verification and Compilation in a Virtual Machine. PhD thesis, University of California, Irvine (2006)

14. Gal, A., Eich, B., Shaver, M., Anderson, D., Kaplan, B., Hoare, G., Mandelin, D., Zbarsky, B., Orendorff, J., Ruderman, J., Smith, E., Reitmair, R., Haghighat, M.R., Bebenita, M., Change, M., Franz, M.: Trace-based just-in-time type specialization for dynamic languages. In: PLDI, pp. 465-478. ACM, New York (2009)

15. Gal, A., Probst, C.W., Franz, M.: Hotpathvm: an effective jit compiler for resourceconstrained devices. In: VEE, pp. 144-153 (2006)

16. Goldberg, D.: What every computer scientist should know about floating-point arithmetic. Comput. Surv. 23, 5-48 (1991)

17. Harrison, W.H.: Compiler analysis of the value ranges for variables. IEEE Trans. Softw. Eng. 3(3), 243-250 (1977)

18. Jones, N.D., Gomard, C.K., Sestoft, P.: Partial Evaluation and Automatic Program Generation, 1st edn. Prentice Hall, Englewood Cliffs (1993)

19. McCarthy, J.: Recursive functions of symbolic expressions and their computation by machine, part i. Communications of ACM 3(4), 184-195 (1960)

20. Patterson, J.R.C.: Accurate static branch prediction by value range propagation. In: PLDI, pp. 67-78. ACM, New York (1995)

21. Rigo, A.: Representation-based just-in-time specialization and the psyco prototype for python. In: PEPM, pp. 15-26. ACM, New York (2004) 
22. Schultz, U.P., Lawall, J.L., Consel, C.: Automatic program specialization for java. TOPLAS 25(4), 452-499 (2003)

23. Shankar, A., Sastry, S.S., Bodík, R., Smith, J.E.: Runtime specialization with optimistic heap analysis. SIG. Not. 40(10), 327-343 (2005)

24. Stephenson, M., Babb, J., Amarasinghe, S.: Bidwidth analysis with application to silicon compilation. In: PLDI, pp. 108-120. ACM, New York (2000)

25. Su, Z., Wagner, D.: A class of polynomially solvable range constraints for interval analysis without widenings. Theoretical Computeter Science 345(1), 122-138 (2005)

26. Guo, S.y., Palsberg, J.: The essence of compiling with traces. In: POPL. ACM, New York (2011) (page to appear)

27. Zaleski, M.: YETI: a gradually extensible trace interpreter. PhD thesis, University of Toronto (2007) 